\title{
Structural evidence for an edge-contaminated vortex phase in a Nb crystal using neutron diffraction
}

\author{
H. A. Hanson, X. Wang, I. K. Dimitrov, ${ }^{*}$ J. Shi, ${ }^{\dagger}$ and X. S. Ling ${ }^{\ddagger}$ \\ Department of Physics, Brown University, Providence RI 02912, USA \\ B. B. Maranville and C. F. Majkrzak \\ NIST Center for Neutron Research, Gaithersburg, MD 20899, USA \\ M. Laver \\ Paul Scherrer Institut, Villigen PSI, CH 5232 Switzerland \\ U. Keiderling and M. Russina \\ Helmholtz Zentrum Berlin fr Materialien und Energie GmbH, 14109 Berlin, Germany \\ (Received 30 April 2011; published 20 July 2011)
}

\begin{abstract}
We report the structural observation of a disordered vortex matter phase existing near the edge of a bulk type-II superconductor $\mathrm{Nb}$ using a novel position-sensitive neutron diffraction technique. This "edge-contaminated" vortex state was implicated in previous studies using transport techniques and magneto-optics and is postulated to play a significant role in the behavior of vortex dynamics in a wide range of type-II superconductors. In this sample, thermal annealing causes the vortex matter in the interior to reorder implying that the edge-contaminated bulk state is metastable. However, the edge vortex structure remains disordered after repeated thermal annealing indicating the spatial coexistence of a vortex glass with a Bragg glass. This observation resolves outstanding issues concerning the peak effect in type-II superconductors.
\end{abstract}

DOI: 10.1103/PhysRevB.84.014506

PACS number(s): 74.25.Uv, 74.78.-w, 78.70.Nx

\section{INTRODUCTION}

Vortex matter in type-II superconductors continues to be a subject of fascination. A longstanding issue is the nature of the ground state of the vortex lines in the presence of atomic impurities and other forms of quenched disorder acting as random pinning centers. ${ }^{1,2}$ Early calculations ${ }^{3,4}$ and scaling arguments ${ }^{5}$ suggested the absence of long-range order even for systems with weak random pinning. Thus, an ordered phase was not expected in any real type-II superconductor contrary to the neutron diffraction experiments in which sharp Bragg peaks were observed from the vortex matter in $\mathrm{Nb}$ samples. ${ }^{6,7}$ A possible reconciliation has been proposed in the Bragg glass model ${ }^{8,9}$ which predicts that the vortex lines form a topologically ordered lattice with quasi-long-range-order (QLRO) characterized by a power-law structure factor. ${ }^{10,11}$ However, the latest theoretical treatments reveal a lack of consensus for the existence of a Bragg glass phase ${ }^{12-14}$ and further experimental progress is required.

The existence of QLRO implies that there must be a true order-disorder phase transition in the vortex matter. A remaining issue is to explain the relationship between this putative phase transition and the peak effect. ${ }^{15-29}$ This effect, a peak in the critical current versus $T$ curves, ${ }^{15}$ often occurs in type-II superconductors with weak bulk pinning. This feature has attracted much attention ${ }^{30,31}$ since it was first observed in $\mathrm{Nb}$ in $1962^{15,16}$ but remains poorly understood. There is clear evidence for a first-order melting transition at the peak effect line from magnetization, ${ }^{21,22}$ neutron diffraction, ${ }^{23}$ and heat capacity ${ }^{28}$ measurements. It has also been observed that the peak can disappear at high or low magnetic fields $27,32,33$ and some samples display neither a peak effect nor any sign of a phase transition. ${ }^{34,35}$ This "lack-of-universality" appears to be related to another seeming contradiction in vortex matter physics: The zero-field-cooled (ZFC) vortex matter state is ordered and the field-cooled (FC) state is disordered in some samples (indicative of supercooling at a first-order transition $)^{23,29,36}$ whereas in other samples, the reverse is true. ${ }^{37}$ There have been strong indications from transport studies ${ }^{38-40}$ that a disordered ZFC vortex matter may be caused by an "edge-contamination" mechanism. ${ }^{41}$ An inhomogeneous surface barrier causes "tearing" 42 to occur as the vortex lines are driven into system from the sample edges. The vortex matter can partially repair itself through the vortex-vortex interactions but the final state may have a coexistence between a disordered edge structure and an ordered interior. The disordered state will trap an excess amount of magnetic flux on the edge of the sample and results in a varying magnetic field profile in the sample. ${ }^{45}$ This model provides an excellent explanation for transport, ${ }^{38,40,43}$ magnetization, ${ }^{44}$ and magneto-optic experiments. ${ }^{45}$ However, there has been no structural evidence for the existence of an edge-contaminated vortex matter phase previously reported due to the limitation of traditional methods of small angle neutron scattering (SANS).

Here we report that a novel neutron diffraction technique finds the vortex matter near the sample edge is indeed disordered in a $\mathrm{Nb}$ crystal exhibiting a disordered ZFC state and a weak peak effect. We show that the bulk ZFC vortex matter structure is metastable and can be thermally annealed into large ordered domains while the edge vortex matter remains disordered. We then oxidize the sample surface to reduce the impact of the inhomogeneous surface barrier. By repeating our neutron diffraction measurements, we find that oxidation reduces the magnetic field profile from the edge to the center of the sample and that the overall structural order of the ZFC vortex matter improves. The FC vortex matter structure should be independent of any edge contamination 


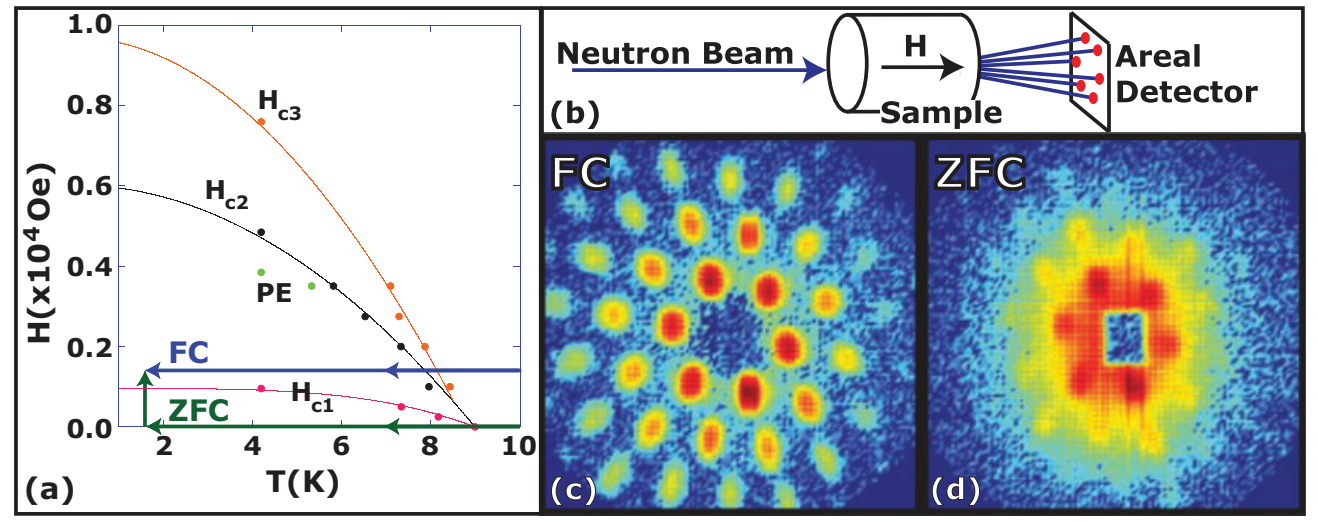

FIG. 1. (Color online) (a) The magnetic field, $H$, versus $T$ phase diagram for the Nb crystal used in this study. PE is remnant of a peak effect. The solid lines are fits to the data. The values of the critical magnetic fields are very similar to a previous $\mathrm{Nb}$ crystal but the peak effect line is very different. ${ }^{27}$ The growth procedures for FC and ZFC are sketched on the diagram. (b) The scattering geometry for conventional small-angle neutron scattering (SANS). (c,d) SANS images, summed over the rocking angle, for the FC and the ZFC states at $T=1.5 \mathrm{~K}$ and $H=1400$ Oe

effect but surprisingly, the FC scattering intensity doubles after surface oxidation. This result suggests that there is another source of disorder in the $\mathrm{Nb}$ crystal that has been changed. We discuss our results in the context of the peak effect and Bragg glass problems.

The article is organized as follows. In Sec. II, we provide the experimental details of the sample, in particular, magnetic field versus temperature phase diagram as measured with AC magnetic susceptibility, and review the basic scattering principles for SANS and our slicing neutron diffraction. In Sec. III, we present our experimental results and discuss possible interpretations. Finally, we summarize our findings and their possible impact on the field of vortex physics in Sec. IV.

\section{EXPERIMENTAL TECHNIQUES}

The $\mathrm{Nb}$ sample is an unpolished as-grown crystal ${ }^{46}$ of $99.99 \%$ purity. It has the shape of a cylinder with the $\langle 111\rangle$ crystallographic direction oriented along its longitudinal axis. The crystal diameter is $12.1 \mathrm{~mm}$ with a height of $10.1 \mathrm{~mm}$ and weighs $9.69 \mathrm{~g}$. It has a residual resistivity ratio of $R R R=\rho_{300 \mathrm{~K}} / \rho_{10 \mathrm{~K}}=32$ and should be an atomic system with weak bulk pinning. From our AC magnetic susceptibility measurements, ${ }^{47}$ we sketch the magnetic phase diagram for this sample in Fig. 1(a). We used a frequency of $1070 \mathrm{~Hz}$ and $H_{a c}=13.5 \mathrm{Oe}$. The onset of superconductivity occurs at $T=9.18 \mathrm{~K}$ and has a width of $400 \mathrm{mK}$. The important sample parameters are: by measurement, the zerofield superconducting transition temperature $T_{c}=9.0 \mathrm{~K}$, and the peak effect $H_{p}(T=4.2 \mathrm{~K})=3850$ Oe; by extrapolation, the zero-temperature critical field values $H_{c 1}=960 \mathrm{Oe}, H_{c 2}=$ $6000 \mathrm{Oe}$, and $H_{c 3}=10000 \mathrm{Oe}$. Our thermo-magnetic growth procedures are defined on the phase diagram: FC is cooling the sample in a magnetic field of $1400 \mathrm{Oe}$ and ZFC is cooling the sample in zero magnetic field and then ramping the field to 1400 Oe.

The SANS data in Fig. 1 are measured using the V4 instrument at the BER II reactor of the Helmholtz Zentrum Berlin. The scattering geometry for the SANS images is shown in Fig. 1(b). The magnetic field, $H$, is applied parallel to the neutron beam and the cylindrical axis of the sample. The neutron wavelength is $\lambda=12 \AA$ and $\Delta \lambda / \lambda=0.1$. Rectangular guides are used for collimation giving an angular spread of $0.146^{\circ}$. The data are summed over the entire rocking curve (the longitudinal direction of the vortex lines).

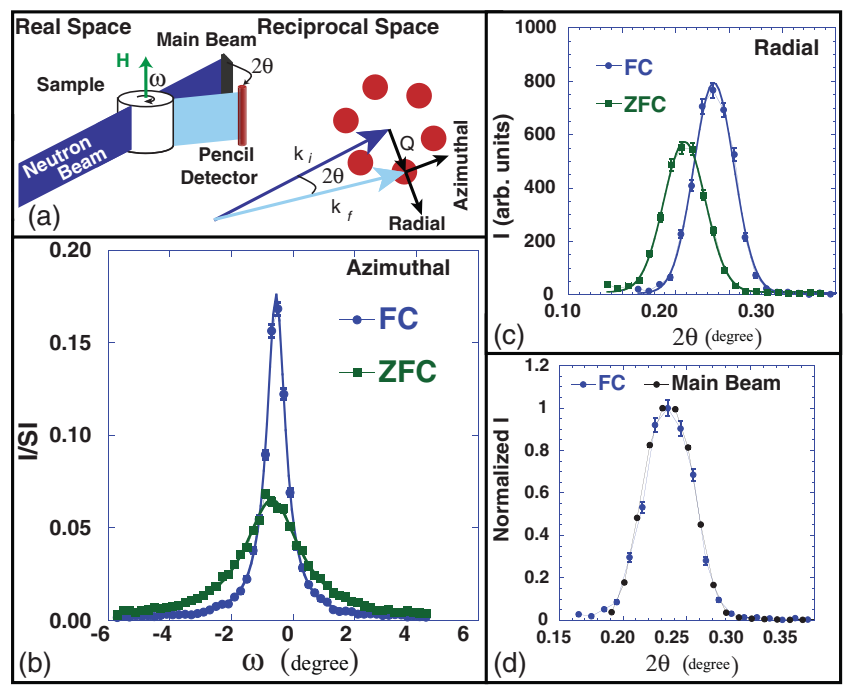

FIG. 2. (Color online) (a) The scattering geometry for AND/R in real space and reciprocal space. Radial scans probe the direction parallel to the reciprocal lattice vector, $Q$, and azimuthal scans measure the direction perpendicular. Note the magnetic field, $H$, is applied parallel to the cylindrical axis of the sample. (b) The scattering intensity as a function of $2 \theta$ plotted for the ZFC and FC vortex matter radial Bragg peaks at $T=3.5 \mathrm{~K}$ and $H=1400$ Oe. Solid lines are Gaussian best fits. (c) The normalized intensity versus $2 \theta$ for the radial FC Bragg peak and the main beam measurement. Note that the FC (and the ZFC) Bragg peak is (are) resolution-limited. (d) The scattering intensity divided by the summed intensity $(I / S I)$ versus $\omega$ for the vortex matter in (b) azimuthal Bragg peaks. Solid lines are Lorentzian fits. Here, neither peak is resolution-limited. The error bars are the statistical standard deviation from the neutron counts. 
At the NIST Center for Neutron Research, we use the Advanced Neutron Diffractometer/Reflectometer (ANDR) ${ }^{48}$ with a scattering geometry as shown in Fig. 2(a). The magnetic field is applied parallel to the $\langle 111\rangle$ axis of the $\mathrm{Nb}$ sample, perpendicular to the neutron beam collimation. This geometry allows us to investigate the radial and azimuthal widths of the in-plane Bragg peak (reciprocal space diagram drawn in Fig. 2(a)) as the sample is scanned by a ribbon-shaped neutron beam. Radial scans probe the direction parallel to the reciprocal lattice vector, $Q$, and azimuthal scans measure the direction perpendicular. A radial scan determines the $2 \theta$ of the Bragg condition, $n \lambda=2 d \sin \theta$ where $n=1$ for the first order Bragg peak and $d$ is the vortex plane spacing determined by the applied magnetic field. Here, the sample is rotated through the Bragg condition in increments of $\Delta \theta$ simultaneously with detector rotations of $2 \Delta \theta$. The azimuthal Bragg peak is measured by rotating the vortex lattice by an angle, $\omega$, and recording the scattered neutron intensity with detector located at the predetermined $2 \theta$. An azimuthal scan reveals to the angular orientation of the vortex matter. The peak width does not correspond to a correlation length but instead a spread in orientations of the vortex lattice planes. Asymmetries in the peak shape reveal the presence of different-sized domains scattering at different values of $\omega$. For ANDR, the incident neutron beam is selected by a monochromator and has a mean wavelength of $\lambda=5 \AA$ and a $\Delta \lambda / \lambda=0.01$. Measurements of the main neutron beam from the slit collimation reveal an angular spread of $0.02^{\circ}$ (Figs. 2,3,6) and $0.009^{\circ}$ (Figs. 4,5).

The AND/R instrument allows us to explore the spatial nature of the disorder in the vortex matter. The neutron beam with a width of 0.5 (or 0.25 ) $\mathrm{mm}$ is much smaller than the sample diameter $(12.1 \mathrm{~mm})$. We are able to vary the section of

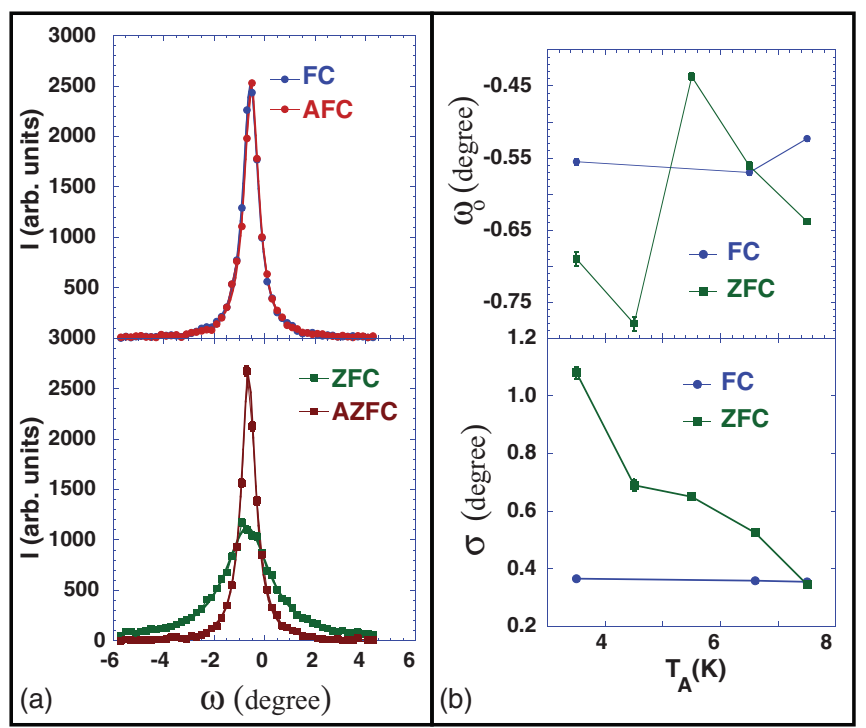

FIG. 3. (Color online) (a) $I$ versus $\omega$ for the annealed Bragg peaks compared to the as-grown Bragg peaks: ZFC (bottom) and FC (top) at $T=3.5 \mathrm{~K}$ and $H=1400$ Oe. Solid lines are Lorentzian fits. (b) The half-width, $\sigma$, (bottom) and Bragg peak center, $\omega_{\circ}$, (top) from the Lorentzian fits at $H=1400$ Oe are plotted as a function of the annealing temperature, $T_{A} . T_{A}$ is the highest temperature the vortex lattice is heated to before returning to the measurement temperature, $T=3.5 \mathrm{~K}$. Solid lines are guides for the eyes.

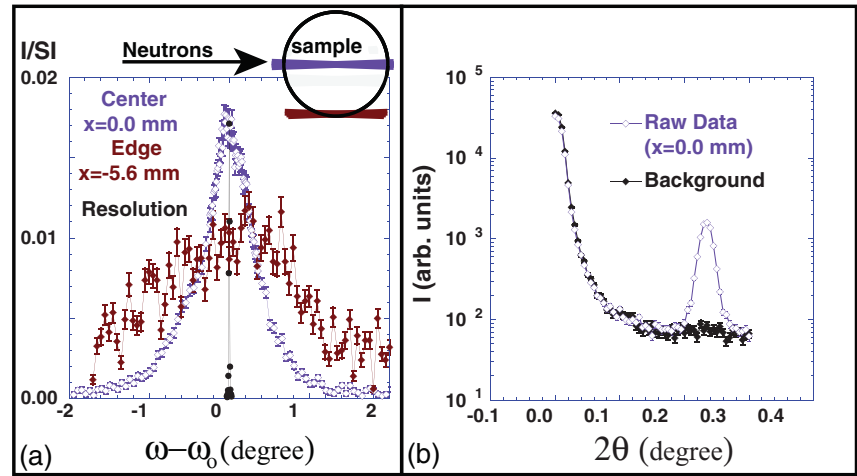

FIG. 4. (Color online) (a, top) A top view of the location of the two Bragg peaks in the $\mathrm{Nb}$ crystal. (a, main) $I / S I$, versus rocking angle minus the location of the Bragg condition, $\omega-\omega_{\circ}$, of the Bragg scattering from the edge of the sample and from the sample center for an annealed FC vortex matter scaled to $4.5 \mathrm{~K}$ and 1400 Oe. The neutron beam measurement is also plotted to show that both peaks are much broader than the instrument resolution. (b) The radial scan of the center Bragg peak and the main beam measurement showing that the center radial peak is resolution-limited.

the $\mathrm{Nb}$ crystal exposed to the neutron beam and measure the Bragg peak for a particular spatial location (the drawn-to-scale sketches of the topography measurements are shown in Figs. 4 and 5). However, the increased resolution is at the expense of neutron flux, and prevents us from studying the peak effect and the order-disorder transition directly. Our scanning neutron diffraction measurements are limited to deep in the Bragg glass portion of the phase diagram. For probing different spatial sections of the vortex matter, we calibrate the center of the $\mathrm{Nb}$ crystal $(x=0.0 \mathrm{~mm})$ via scans of a neutron absorber $(\mathrm{Cd}$ mask) located on the bottom of the sample. The $\mathrm{Cd}$ mask allows us to identify the location of two edges of the cylinder in the neutron beam coordinate system.

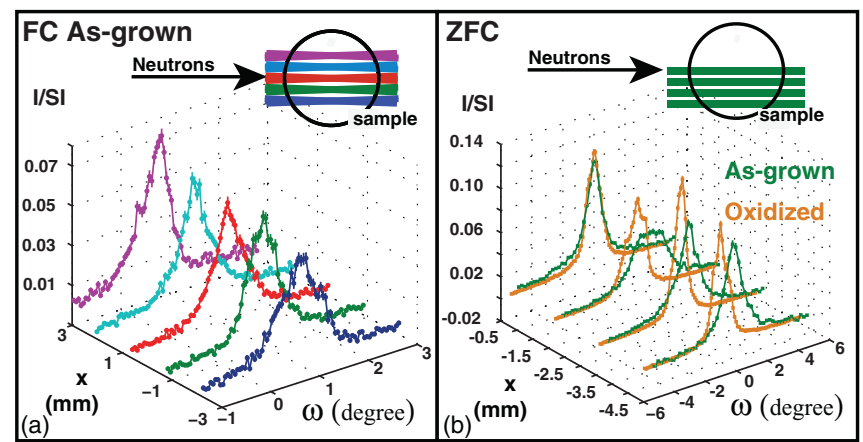

FIG. 5. (Color online) (a, top) The scattering location for the five Bragg peaks from an AFC vortex lattice. (a, main) 3D plot of the AFC Bragg peaks at $4.5 \mathrm{~K}$ and 1400 Oe: $I / S I$ versus $\omega$ versus sample position, $x$, with a top view of the respective spatial positions in the $\mathrm{Nb}$ crystal. The solid lines are guides for the eyes. (b, top) The scattering location in the $\mathrm{Nb}$ crystal. Notice here the scattering moves from the center to one edge. (b, main) 3D plot of the ZFC Bragg peaks for the as-grown $\mathrm{Nb}$ sample compared to the surface oxidized sample at $4.5 \mathrm{~K}$ and 1400 Oe: $I / S I$ versus $\omega-\omega_{\circ}$ versus $x$. The peaks are all shifted by the same $\omega_{\circ}$ for each history: The centers of the center Bragg peaks is shifted to zero. The solid lines are guides for the eyes. 


\section{RESULTS AND DISCUSSION}

\section{A. The peak effect problem and disordered ZFC vortex matter}

The AC magnetic susceptibility phase diagram in Fig. 1(a) is very similar to a previous $\mathrm{Nb}$ crystal diagram ${ }^{27}$ but there are two notable differences between the samples. First, this $\mathrm{Nb}$ crystal is even cleaner than the previous sample which had an $R R R=14 .{ }^{33}$ Second, the previous sample displayed a pronounced peak effect which was observable (using AC magnetic susceptibility) ${ }^{18}$ down to 800 Oe..$^{27}$ In this sample, a remnant of the peak effect occurs in the temperature-dependent AC susceptibility measurements at fields above 3400 Oe, and a weak peak effect occurs at $4.2 \mathrm{~K}$ and $3850 \mathrm{Oe}$. We use neutron diffraction techniques to untangle the vortex physics which may account for the different peak effect behavior in these seemingly similar samples.

Figs. 1(c) and 1(d) demonstrate the drastic differences in structural order between the FC and ZFC vortex matter. The FC SANS image shows well defined Bragg peaks for $n=1, \ldots, 4$ confirming that the FC vortex matter is ordered. This result is unsurprising because though the FC growth procedure crosses a disordered phase of the vortex matter, we expect little supercooling effects ${ }^{23,36}$ as the region we study is the low-field/low-temperature portion of the phase diagram. For the ZFC state, the Bragg peaks are emerging but significant scattering occurs between the expected peaks indicating a disordered vortex matter. The presence of a disordered ZFC structure combined with the weak peak effect behavior makes this sample an interesting candidate for studying the edge contamination model. However, due to the present technical limitation of most SANS instruments, it is difficult to characterize the spatial variation of the vortex matter structure. In the next section, we show that one can overcome this difficulty by using a neutron reflectometer in the diffraction mode.

\section{B. Slicing neutron diffraction results}

Our experiment is made possible by a reflectometry instrument $^{48}$ which has the novel capability of neutron diffraction topography. ${ }^{49}$ This technique allows positionaldependent structural analysis of a bulk vortex state in a type-II superconductor. Unlike SANS measurements where a position-sensitive detector provides information on the inplane Bragg peak, one ANDR scan is required for locating $2 \theta$ (radial) and a second for studying the vortex lattice orientation (azimuthal). Figure 2(b) is a radial scan of the intensity, $I$, versus $2 \theta$ for the FC and ZFC states. Since $d=\lambda /(2 \sin \theta)$, the different $2 \theta$ values reveal the expected magnetic field profile for a sample with edge contamination. The ZFC $2 \theta$ is smaller than the FC one, which corresponds to $d_{\mathrm{ZFC}}>d_{\mathrm{FC}}$ and implies that the vortex lines are packed less densely in the center of the ZFC vortex matter. The main beam measurement is plotted against the FC Bragg peak in Fig. 2(c) and reveals that the scattered peaks are resolution-limited as expected for a Bragg glass. ${ }^{11}$ We find that all radial measurements are resolution-limited, unlike the ZFC SANS results. The difference between the ANDR and SANS ZFC measurements is the amount of the sample illuminated by the neutron beam. In the SANS experiment, approximately $43 \%$ of sample volume is being measured to determine the interior bulk behavior and the scattering corresponds to a disordered structure. In ANDR, we are probing a narrow section of the vortex matter, around $11 \%$ of the $\mathrm{Nb}$ crystal, and only a small number of domains meet the Bragg condition at this specific angle. The fact that the $\mathrm{ZFC}$ radial peak is resolution-limited suggests that there are very few structural defects inside each vortex lattice domain affecting the planar spacing.

Although both the ZFC and FC radial peaks are resolutionlimited, the azimuthal Bragg peak structure shown in Fig. 2(d) is very different. For this measurement, we plot scattering intensity divided by the summed intensity, $I / S I$, versus $\omega$. Here, unlike the FC SANS measurement in Fig. 1(b), the width of the azimuthal FC Bragg peak is not resolution-limited. It is clear that $\sigma_{\mathrm{FC}}<\sigma_{\mathrm{ZFC}}$ where $\sigma$ is the Lorentzian half-width of the peak and that $I_{\text {FCpeak }}>I_{\text {ZFCpeak }}$ where $I_{\text {peak }}$ is the peak intensity of the scattering. These are the same general features as found in the SANS data but with additional fine structure information. An asymmetry in the ZFC peak is now visible, which combined with the peak broadness and the resolutionlimited radial peak, suggest a vortex lattice broken into many small domains separated by low-angle grain boundaries. This spread in vortex lattice orientation is interpreted as a disordered structure. The sharp FC peak is expected but the FC width is much larger than the instrument resolution. This spread in angular orientation suggests that the bulk pinning of the $\mathrm{Nb}$ crystal is inducing a deformation in the $\mathrm{FC}$ vortex lattice structure.

We find that the disordered ZFC state is metastable and will reorder upon thermal cycling. Our procedure is to heat the as-grown vortex lattice in increments of $\Delta T=0.5 \mathrm{~K}$, holding for one minute at each temperature until $T_{A}$ is reached. $T_{A}$ is held for two minutes before recooling in increments of $\Delta T=1.0 \mathrm{~K}$ back to $T=3.5 \mathrm{~K} . T_{A}$ is defined as the highest stable temperature the vortex matter attains before returning to the measurement temperature, $T=3.5 \mathrm{~K}$. In Fig. 3(a) the Bragg peaks from before and after thermally cycling the vortex matter to $T_{A}=7.5 \mathrm{~K}$ are plotted for the FC (top) and ZFC (bottom) histories. Thermal annealing has little impact on the FC vortex matter; there is a slight decrease in peak width and increase in peak intensity. However, it is clear from Fig. 3(a) that the ZFC vortex matter undergoes substantial restructuring with increased thermal energy. In Fig. 3(b), the Lorentzian half-widths and the centers of the Bragg peaks are plotted as a function of $T_{A}$. All measurements in Fig. 3 are made at the same $H=1400$ Oe and $T=3.5 \mathrm{~K}$. It is interesting to note that the location of $2 \theta$ also changes with thermal annealing. After $T_{A}>4.5 \mathrm{~K}$, the peaks occur at the same $2 \theta$ (data not shown). This smoothing of the field profile suggests that the thermal annealing procedure is allowing the system to relax to its equilibrium state. ${ }^{50}$ The rotation of the ZFC Bragg peak center with thermal cycling suggests a recrystallization process where different grains are absorbed by others. The orientation of the largest grain will determine the center of the azimuthal Bragg peak and the shifting of the center with thermal energy implies different grains are growing and perhaps rotating as the system cycles. There is a slight plateau effect at $T_{A}=5.5 \mathrm{~K}$ which may indicate two types of reordering processes. This flattening becomes more pronounced when smaller steps in $T_{A}$ are made. To within our experimental resolution, the annealed ZFC and 
FC histories have qualitatively similar structural order with slightly different angular orientations.

Our next step is to use ANDR to characterize the spatial variation of the annealed vortex matter phase. Figure 4(a) shows the azimuthal Bragg peaks from the center and edge of the sample for an annealed FC state (with the instrument resolution) while Fig. 4(b) is the radial measurement of the center vortex matter. In the azimuthal measurements, a sharp asymmetric peak is found for the center of the sample indicating an ordered vortex matter phase. Upon a close examination, one can discern fine structures indicating large ordered domains of vortex matter separated by low angle grain boundaries. The resolution-limited radial measurement causes us to interpret this state as the Bragg glass phase with residual nonequilibrium effects. The radial correlation length ${ }^{37}$ as calculated from our instrument resolution is $\xi \approx 584 a$ where $a$ is the vortex lattice spacing. The broad Bragg peak at the edge of the sample suggests a disordered vortex matter phase persisting even after thermal annealing. The radial data for edge state has significantly lower scattering but is still resolution-limited. The low intensity radial scattering supports the model that the edge state is broken into many small domains and these domains scatter at different orientations resulting in the broad noisy peak in Fig. 4(a). We suggest that this may be a vortex glass phase ${ }^{51}$ co-existing on the sample edge. Figure 4(a) is structural evidence for the edge contamination model.

Figure 4(a) shows that the disordered edge vortex matter exists even in the annealed state. This observation is significant because it implies that the structural information obtained by standard SANS measurements is not directly correlated with dynamical measurements such as magnetization and transport for samples with edge contamination (with an inhomogeneous surface barrier). The SANS signal is dominated by the central region of the sample, while the latter are determined by supercurrent or magnetic field gradients on the sample surface. Furthermore, our observations offer a resolution to the longstanding puzzle concerning the appearance or absence of the peak effect. By using AC magnetic susceptibility measurements, we find that in this sample, the peak-effect line has a significantly lower slope than that of $H_{c 2}(T)$. In the previous sample, the line is nearly parallel to the $H_{c 2}(T)$ line. Because the AC magnetic susceptibility measurements are dominated by the surface current in the sample, the appearance or the absence of the peak effect will depend on the residual shortrange order in the edge vortex matter. If the edge vortex matter is strongly disordered by the surface defects, there will be no observable effect from the thermally-induced disordering at the Bragg glass melting transition in the bulk. In systems with minimal contamination effects, the peak effect is pronounced and coincides with the Bragg glass melting transition measured with SANS. ${ }^{23}$ An earlier observation that the peak effect in $2 \mathrm{H}-\mathrm{NbSe}_{2}$ can be lowered (in field for fixed $T$ measurement) by physically cutting the sample ${ }^{52}$ may also be explained by the presence of a dirty vortex state at the sample edges.

In Fig. 5(a), we measure the variation of the Bragg peak structure through the interior of the sample. As the neutron beam moves across the sample, there are clear changes in the structure of the Bragg peak. These variations indicate a complex domain structure in the vortex matter, consistent with previous Reverse Monte Carlo Refinements calculated for SANS data in another Nb sample. ${ }^{53}$

\section{Impact of surface oxidation}

Surface oxidation has been shown to suppress the surface barrier effects in $\mathrm{Nb}{ }^{54,55}$ To carry out a preliminary study of the surface oxidation impact on the vortex matter, we subject our sample to an $\mathrm{O}_{2}$ atmosphere at $600^{\circ} \mathrm{C}$ for six minutes following the recipe by Ref. 55. After oxidation, we repeat our neutron diffraction measurements. A subscript $o$ is added to the growth procedure to denote the oxidized sample. Figure 5(b) is a 3D plot of the azimuthal Bragg peaks versus $x$ and $\omega$ for ZFC vortex matter before and after oxidation. An observant reader might notice that the Bragg peak in the center of the sample from the as-grown ZFC state is narrower with higher intensity than the ZFC Bragg peak in Fig. 2(d). We find that the ZFC Bragg peak changes every time the vortex lattice is formed. We conjuncture that this behavior is because of the dynamic nature of the edge contamination effect. Nevertheless, it is clear that the structural order in the system has increased after oxidation through the higher intensity and narrower widths of all the $\mathrm{ZFC}_{o}$ Bragg peaks as compared with the as-grown ones.

Focusing on the impact of surface oxidation on the vortex matter in the interior of the sample, we plot the center Bragg peaks from before and after oxidation in Fig. 6(a) $(I / S I$ versus $\omega-\omega_{\circ}$ ) for the FC (top) and ZFC (bottom) vortex matter. The ZFC Bragg peak has strong variations in the tail, a broader width, and a lower peak intensity. The broad tails are attributed to a strongly disordered edge state that the neutron beam bisects due to the ANDR scattering geometry.

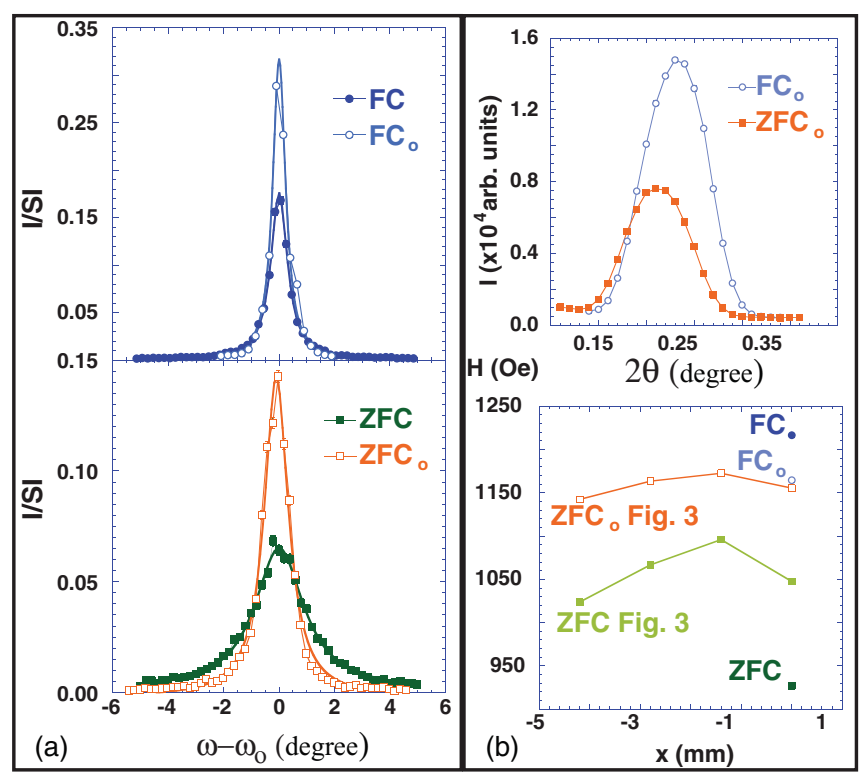

FIG. 6. (Color online) The impact of surface oxidation on the FC and ZFC vortex matter is shown. (a) The intensity divided by the summed intensity $(I / S I)$ is plotted versus $\omega-\omega_{\circ}$ for the FC versus $\mathrm{FC}_{o}$ vortex matter (top) and ZFC versus $\mathrm{ZFC}_{o}$ vortex matter (bottom). (b) The radial scans of $\mathrm{FC}_{o}$ and $\mathrm{ZFC}_{o}$ in the center of the $\mathrm{Nb}$ crystal are shown. (top). The $B$ value is plotted versus the sample position, $x$, for ZFC and FC vortex matter(bottom). The measurements are at $T=4.5 \mathrm{~T}$ and $H=1400$ Oe. 
The change in the tail behavior suggests that the order in the vortex matter near the sample edge has increased after the oxidation of the cylindrical surface. This model is supported by Fig. 5(b) where the largest difference between peak structure is nearest to the sample edge. The increase in relative intensity and decrease in width for the $\mathrm{ZFC}_{o}$ vortex matter is attributed to an improved order in the bulk vortex structure. Comparing $\mathrm{FC}$ to $\mathrm{FC}_{o}$, we see a higher intensity in the $\mathrm{FC}_{o}$ Bragg peak with minor scattering differences in the tails. Since the FC vortex matter structure formation should be independent of any edge contamination mechanism, we suggest that this intensity change is actually due to the underlying atomic structure of the $\mathrm{Nb}$ crystal and the oxidation of the top and bottom surfaces. Because this sample is unpolished, we suspect that the rough surfaces were acting as pinning sites for the vortex lines. Surface oxidation will reduce the pinning on the entire exterior and appears to greatly increase the order of the bulk system. It is important to note that because surface oxidation leads to diffusion, a perfectly uniform surface barrier can never be formed and it is not surprising that $\mathrm{ZFC}_{o}$ state is still disordered with respect the the $\mathrm{FC}_{o}$ vortexmatter.

To isolate the effect of surface oxidation on the field profile of the system, the radial scans from the $\mathrm{FC}_{o}$ and $\mathrm{ZFC}_{o}$ vortex lattices in the sample center are shown in Fig. 6(b, top). The difference in $2 \theta$ between the two histories has greatly decreased when compared to Fig. 2(b). To quantify this change in the radial measurements, Fig. 6 (b, bottom) has a plot of $\mathrm{B}$ versus $x$ where $B$ was calculated using the formula, $B=\lambda^{2} \sqrt{3} \Phi_{\circ} /\left(16 \pi^{2} \sin ^{2} \theta\right)\left(\Phi_{\circ}\right.$ is the quantum fluxoid). The field profile of the ZFC vortex matter varies by $120 \mathrm{G}$ in the sample center and $\approx 75 \mathrm{G}$ through the sample. In comparison, the $\mathrm{ZFC}_{o}$ field values varies by only $30 \mathrm{G}$ throughout the sample. The $\mathrm{FC}_{o} 2 \theta$ is different from the $\mathrm{FC}$ one by about $50 \mathrm{G}$ so the largest changes occur between the $\mathrm{ZFC}$ and $\mathrm{ZFC}_{o}$ vortex matter.

\section{CONCLUSIONS}

In summary, using a slicing neutron diffraction approach, we have explored the vortex matter in a type-II superconductor with a disordered ZFC vortex matter and weak peak effect behavior. Our data offer structural evidence that an edge contamination mechanism is indeed present in systems with a disordered ZFC vortex state. We find resolution-limited radial Bragg peaks for all the thermal-magnetic histories but very different behavior in the azimuthal peaks. A thermal annealing procedure reveals the metastability of the ZFC vortex matter structure and a slight increase in order for the FC system. The surface oxidation of the Nb crystal shows the expected suppression of the magnetic field profile from edge contamination as well as an overall increase in order for all vortex matter histories. The presence of edge contamination in our system could explain the lack of universal behavior for the peak effect in seemingly similar Nb samples. Our results shed light on the phase diagrams for type-II superconductors and may offer a route to the growth of a true Bragg glass state.

\section{ACKNOWLEDGMENTS}

This research was supported by the US Department of Energy, Office of Basic Energy Sciences, Division of Materials Sciences and Engineering under grant DE-FG02 07ER46458. The ANDR measurements were supported by the Department of Commerce. The SANS measurements were supported by the European Commission under the $7^{\text {th }}$ Framework Programme through "Research Infrastructures" action of the "Capacities" Programme, contract number CP-CSA INFRA-2008 - 1.1.1. Number 226507-NMI3. H.A.H. and X.W. would like to acknowledge support from the Galkin Fund at Brown University.
*Current address: Brookhaven National Laboratories, Upton, NY 11973, USA

${ }^{\dagger}$ Permanent address: School of Physics, Wuhan University, Wuhan 430072, PRC

${ }^{\ddagger}$ xsling@brown.edu

${ }^{1}$ T. Giamarchi and S. Bhattacharya, in "High Magnetic Fields: Applications in Condensed Matter Physics and Spectroscopy", ed. C. Berthier et al. (Springer-Verlag, 2002) p. 314.

${ }^{2}$ B. Rosenstein and Dingping Li, Rev. Mod. Phys. 82, 109 (2010).

${ }^{3}$ A. I. Larkin, Sov. Phys. JETP 31, 784 (1970).

${ }^{4}$ A. I. Larkin and Yu. N. Ovchinnikov, J. Low Temp. Phys. 34, 409 (1979).

${ }^{5}$ Y. Imry and S. Ma, Phys. Rev. Lett. 35, 1399 (1975).

${ }^{6}$ J. Schelten, H. Ullmaier, and W. Schmatz, Phys. Status Solidi 48, 619 (1971).

${ }^{7}$ D. K. Christen, F. Tasset, S. Spooner, and H. A. Mook, Phys. Rev. B 15, 4506 (1977).

${ }^{8}$ T. Nattermann, Phys. Rev. Lett. 64, 2454 (1990).

${ }^{9}$ T. Giamarchi and P. Le Doussal, Phys. Rev. Lett. 72, 1530 (1994).

${ }^{10}$ T. Giamarchi and P. Le Doussal, Phys. Rev. B 52, 1242 (1995).
${ }^{11}$ T. Klein, I. Joumard, S. Blanchard, J. Marcus, R. Cubitt, T. Giamarchi, and P. Le Doussal, Nature 413, 404 (2001).

${ }^{12}$ M. Tissier and G. Tarjus, Phys. Rev. Lett. 96, 087202 (2006).

${ }^{13}$ P. Le Doussal and K. J. Wiese, Phys. Rev. Lett. 96, 197202 (2006).

${ }^{14}$ R. Fisch, Phys. Rev. B 79, 214429 (2009).

${ }^{15}$ S. H. Autler, E. S. Rosenblum, and K. Gooen, Phys. Rev. Lett. 9, 489 (1962).

${ }^{16}$ W. DeSorbo, Rev. Mod. Phys. 36, 90 (1964).

${ }^{17}$ P. H. Kes and C. C. Tsuei, Phys. Rev. B 28, 5126 (1983).

${ }^{18}$ X. S. Ling and J. I. Budnick, in Magnetic Susceptibility of Superconductors and Other Spin Systems, R. A. Hein et al. (Plenum Press, New York, 1991), p. 377.

${ }^{19}$ W. K. Kwok, J. A. Fendrich, C. J. van der Beek, and G. W. Crabtree, Phys. Rev. Lett. 73, 2614 (1994).

${ }^{20}$ T. Ishida, K. Okuda, and H. Asaoka, Phys. Rev. B 56, 5128 (1997).

${ }^{21}$ J. Shi, X. S. Ling, Ruixing Liang, D. A. Bonn, and W. N. Hardy, Phys. Rev. B 60, R12593 (1999).

${ }^{22}$ T. Nishizaki, K. Shibataa, T. Sasakia, and N. Kobayashi, Physica C 341-348, 957 (2000). 
${ }^{23}$ X. S. Ling, S. R. Park, B. A. McClain, S. M. Choi, D. C. Dender, and J. W. Lynn, Phys. Rev. Lett. 86, 712 (2001).

${ }^{24}$ M. Marchevsky, M. Higgins, and S. Bhattacharya, Nature 409, 591 (2001).

${ }^{25}$ A. M. Troyanovski, M. van Hecke, N. Saha, J. Aarts, and P. H. Kes, Phys. Rev. Lett. 89, 147006 (2002).

${ }^{26}$ M. Pissas, S. Lee, A. Yamamoto, and S. Tajima, Phys. Rev. Lett. 89, 097002 (2002).

${ }^{27}$ S. R. Park, S. M. Choi, D. C. Dender, J. W. Lynn, and X. S. Ling, Phys. Rev. Lett. 91, 167003 (2003).

${ }^{28}$ R. Lortz, F. Lin, N. Musolino, Y. Wang, A. Junod, B. Rosenstein, and N. Toyota, Phys. Rev. B 74, 104502 (2006).

${ }^{29}$ G. Pasquini, D. Prez Daroca, C. Chiliotte, G. S. Lozano, and V. Bekeris, Phys. Rev. Lett. 100, 247003 (2008).

${ }^{30}$ P. W. Anderson and Y. B. Kim, Rev. Mod. Phys. 36, 39 (1964).

${ }^{31}$ A. B. Pippard, Philos. Mag. 19, 217 (1969).

${ }^{32}$ X. S. Ling, J. I. Budnick, and B. W. Veal, Physica C 282, 2191 (1997).

${ }^{33}$ N. D. Daniilidis, I. K. Dimitrov, V. F. Mitrović, C. Elbaum, and X. S. Ling, Phys. Rev. B 75, 174519 (2007).

${ }^{34}$ E. M. Forgan, S. J. Levett, P. G. Kealey, R. Cubitt, C. D. Dewhurst, and D. Fort, Phys. Rev. Lett. 88, 167003 (2002).

${ }^{35}$ C. J. Bowell, R. J. Lycett, M. Laver, C. D. Dewhurst, R. Cubitt, and E. M. Forgan, Phys. Rev. B 82, 144508 (2010).

${ }^{36}$ Z. L. Xiao, O. Dogru, E. Y. Andrei, P. Shuk, and M. Greenblatt, Phys. Rev. Lett. 92, 227004 (2004).

${ }^{37}$ U. Yaron, P. L. Gammel, D. A. Huse, R. N. Kleiman, C. S. Oglesby, E. Bucher, B. Batlogg, D. J. Bishop, K. Mortensen, K. Clausen, C. A. Bolle, and F. De La Cruz, Phys. Rev. Lett. 73, 2748 (1994).

${ }^{38}$ Y. Paltiel, D. T. Fuchs, E. Zeldov, Y. N. Myasoedov, H. Shtrikman, M. L. Rappaport, and E. Y. Andrei, Phys. Rev. B 58, R14763 (1998).

${ }^{39}$ Y. Paltiel, E. Zeldov, Y. Myasoedov, M. L. Rappaport, G. Jung, S. Bhattacharya, M. J. Higgins, Z. L. Xiao, E. Y. Andrei,
P. L. Gammel, and D. J. Bishop, Phys. Rev. Lett. 85, 3712 (2000).

${ }^{40}$ Z. L. Xiao, E. Y. Andrei, Y. Paltiel, E. Zeldov, P. Shuk, and M. Greenblatt, Phys. Rev. B 65, 094511 (2002).

${ }^{41}$ Y. Paltiel, E. Zeldov, Y. N. Myasoedov, H. Shtrikman, S. Bhattacharya, M. J. Higgins, Z. L. Xiao, E. Y. Andrei, P. L. Gammel, and D. J. Bishop, Nature 403, 398 (2000).

${ }^{42}$ S. Bhattacharya and M. J. Higgins, Phys. Rev. B 52, 64 (1995).

${ }^{43}$ M. Reibelt, A. Schilling, and N. Toyota, Phys. Rev. B 81, 094510 (2010).

${ }^{44}$ H. Küpfer, G. Ravikumar, Th. Wolf, A. A. Zhukov, and R. MeierHirmer, Phys. Rev. B 70, 144509 (2004).

${ }^{45}$ D. Giller, A. Shaulov, T. Tamegai, and Y. Yeshurun, Phys. Rev. Lett. 84, 3698 (2000).

${ }^{46}$ The crystal was commercially grown by Goodfellow Ltd. UK.

${ }^{47}$ H. Hanson Ph. D. Thesis Brown University 2011.

${ }^{48}$ J. A. Dura, D. J. Pierce, C. F. Majkrzak, N. C. Maliszewskyj, D. J. McGillivray, M. Lösche, K. V. ODonovan, M. Mihailescu, U. Perez-Salas, D. L. Worcester, and S. H. White, Rev. Sci. Instrum. 77, 074301 (2006).

${ }^{49}$ M. Schlenker, J. Baruchel, R. Perrier de la Bâthie, and S. A. Wilson, J. Appl. Phys. 46, 2845 (1975).

${ }^{50}$ D. Giller, B. Ya. Shapiro, I. Shapiro, A. Shaulov, and Y. Yeshurun, Phys. Rev. B 63, 220502R (2001).

${ }^{51}$ D. S. Fisher, M. P. A. Fisher, and D. A. Huse, Phys. Rev. B 43, 130 (1991).

${ }^{52}$ X. S. Ling, S. J. Smullin, J. E. Berger, W. L. Karlin, D. E. Prober, and R. Liang, Philos. Mag. Lett. 79, 399 (1999).

${ }^{53}$ M. Laver, E. M. Forgan, A. B. Abrahamsen, C. Bowell, Th. Geue, and R. Cubitt, Phys. Rev. Lett. 100, 107001 (2008).

${ }^{54}$ S. T. Sekula and R. H. Kernohan, Phys. Rev. B 5, 904 (1972).

${ }^{55}$ G. P. Van Der Mey, P. H. Kes, and D. de Klerk, Physica B \& C 95, 369 (1978).

${ }^{56}$ C. P. Bean, Phys. Rev. Lett. 8, 250 (1962). 